\title{
INCEPTION: is a larger trial to evaluate intraoperative renal replacement therapy in liver transplant patients more than just a dream?
}

\author{
Edward G. Clark, MD (D) Swapnil Hiremath, MD, MPH
}

Received: 4 July 2019/Accepted: 4 July 2019/Published online: 24 July 2019

(C) Canadian Anesthesiologists' Society 2019

The perioperative management of liver transplant (LT) patients is complicated by multiple factors. Liver transplant patients are frequently hemodynamically unstable with metabolic derangements, coagulopathy, and multiorgan failure, including acute kidney injury (AKI). ${ }^{1-3}$ The surgery itself may exacerbate these issues with significant bleeding, graft reperfusion, and large volume intravenous fluid infusion leading to rapid changes in acid-base status (e.g., acidosis), serum electrolyte concentrations (e.g., hyperkalemia and hyponatremia), and hypotonicity. The associated hemodynamic instability and brain edema (with increased intracranial pressure) can result in complications such as arrhythmia, central pontine myelinolysis, brain stem herniation, and graft failure-not the intended end result of a surgery meant to restore organ function., ${ }^{4,5}$

Many centres have now reported that intraoperative renal replacement therapy (IoRRT) during LT may reduce the incidence of these potential complications. ${ }^{6-10}$ Various modalities of renal replacement therapy (RRT) have been used for IoRRT in this context. ${ }^{11}$ Nevertheless, IoRRT is expensive, ${ }^{7,12}$ requires local expertise, and itself may be associated with complications, such as those related to temporary hemodialysis catheter insertion (e.g., infection, pneumothorax), continuous renal replacement therapy (CRRT)-related metabolic derangements (e.g., hypo- or hypercalcemia, hypophosphatemia), and resulting arrhythmias. ${ }^{13}$ Thus, it remains uncertain if IoRRT

E. G. Clark, MD $(\bowtie) \cdot$ S. Hiremath, MD, MPH

The Ottawa Hospital and University of Ottawa, Riverside Campus, 1967 Riverside Drive, Ottawa, ON K1H 7W9, Canada e-mail: edclark@toh.ca

Kidney Research Centre, Ottawa Hospital Research Institute, Ottawa, ON, Canada should be routinely used during LT, and if so, for which patients. It is intuitive that more severely ill patients with pre-existing AKI would be the most likely to benefit from IoRRT. In particular, patients who already require RRT prior to LT might benefit from avoiding a "post-dialysis rebound" phenomenon (exacerbating acidosis and hyperkalemia) due to interruption of RRT prior to LT. ${ }^{6}$

In this issue of the Journal, Karvellas et al. at the University of Alberta report the results of a single-centre pilot randomized-controlled trial of intraoperative continuous renal replacement therapy (IoCRRT) for patients undergoing LT (the intraoperative continuous renal replacement therapy during liver transplantation [INCEPTION] trial). ${ }^{14}$ Patients were included if they had at least mild renal impairment at baseline either due to AKI (RIFLE $R$ category, or worse) or due to chronic kidney disease (estimated glomerular filtration rate $<60$ $\left.\mathrm{mL} \cdot \mathrm{min}^{-1} \cdot 1.73 \mathrm{~m}^{-2}\right)$. After 60 patients had been entered, $32(53 \%)$ of whom were randomized to either IoCRRT or standard-of-care (SOC) treatment, the study was stopped because of slow enrollment and a lack of continued funding. Of the 17 patients randomized to SOC, seven (41\%) crossed over to receive IoCRRT. Overall, no adverse events were reported to have occurred secondary to IoCRRT itself, and there were no significant differences between the groups in terms of clinical outcomes (which this study did not seek to directly address as it was underpowered to do so). Overall, one-year survival was excellent (92\% after excluding those who did not receive a LT following randomization). The authors concluded that a large multicentre trial of IoCRRT would be feasible and safe. $^{14}$

Obtaining better evidence regarding the application of IoCRRT for LT by means of a large, randomizedcontrolled trial is a worthwhile endeavour-if effective, 
broader use of IoCRRT has the potential to improve patient outcomes. If not effective, reduced use would, at minimum, lead to significantly decreased healthcare costs. ${ }^{7}$ A brief review of recent studies assessing the use of pre-LT RRT and/or IoCRRT ${ }^{7-9,15-17}$ (Table) reveals wide variation with respect to the frequency with which IoCRRT is used for LT patients. Importantly, it suggests that the use (or non-use) of IoCRRT in patients with or without significant renal dysfunction preoperatively falls within the current SOC. Differing rates of use may, to some extent, reflect patient case mix but also are likely related to differing local resources and experience with IoCRRT. The current degree of practice variation for the application of this complex treatment (IoCRRT) suggests that there is a strong argument that randomization of LT patients with at least mild kidney dysfunction to either IoCRRT or not should be acceptable to most clinicians despite any strongly held beliefs about its utility in the absence of higher level evidence than currently exists. Nonetheless, this also highlights what may be a major challenge to performing a larger multicentre trial. Despite differing conclusions as to the efficacy of IoCRRT for LT patients, recent singlecentre studies assessing the use of preoperative RRT and/or IoCRRT (Table) generally concluded that the practices at their own study centre constituted the most appropriate management. Although this phenomenon is unlikely to be unique to this particular topic of study, it does suggest that clinicians may already have strong beliefs with respect to the effects of IoCRRT for LT and which patient groups are not clearly going to benefit or be harmed according to whether they receive IoCRRT. This results in a scenario where the totality of the evidence suggests clinical equipoise exists, but that individual centres may not agree that equipoise exists. (i.e., some centres would not be accepting of randomizing their patients to IoRRT or not).

Many aspects of the INCEPTION results are helpful when it comes to planning a larger definitive trial. It is notable that there was significant crossover to IoCRRT from the SOC arm in INCEPTION (41\%). ${ }^{14}$ The potential for large numbers of patients crossing over from SOC to IoCRRT would have to be factored into sample size calculations for a larger trial as a hindrance to enrolling adequate numbers for a suitably powered intention-to-treat analysis. This might also reflect lack of equipoise for randomizing patients in whom most physicians might feel IoCRRT is required. Hence, such patients may not only be difficult to enrol but the risk of contamination may make the effort to enrol such patients unwise. To compensate, one could consider including patients with lower Model for End-Stage Liver Disease (MELD) scores (the mean MELD score in INCEPTION was 36). Nevertheless, these patients are less likely to require (and therefore benefit from)
IoCRRT in the first place and may, in fact, be more likely to be harmed by unnecessary RRT. ${ }^{18}$ It is important to remember that dialysis-catheter-related complications and other complications of CRRT (e.g., hemodynamic instability during RRT [HIRRT]), have the potential to negatively impact outcomes. ${ }^{19}$ The smaller than desired numbers enrolled in this pilot trial is not entirely surprising and provide a more realistic estimate of the number of centres and time needed to conduct a definitive trial. Overall, it is encouraging that the pilot trial results are very reassuring with respect to safety outcomes: no adverse events secondary to IoCRRT were reported. At the same time, the University of Alberta group has extensive expertise in the use of IoCRRT that may have led to fewer adverse events than might occur in a larger multicentre trial, and this safety outcome may not be generalizable to other centres. As an example, members of this group previously reported a quality assurance study which successfully minimized overly rapid perioperative sodium rises in LT patients. ${ }^{20}$ Specifically, they developed and introduced standardized orders for starting and adjusting IoCRRT during LT. These standardized orders were used to specifically address appropriate modification of RRT fluids to prevent overly rapid hyponatremia correction in LT patients and might have prevented adverse events related to overly rapid correction of hyponatremia with IoCRRT.

A more general related challenge to any trial assessing the application of CRRT in critically ill patients is the heterogeneity in CRRT practice ${ }^{21}$ and the lack of validated benchmarks for CRRT quality. ${ }^{22,23}$ Variation in the application of IoCRRT across centres (e.g., use of regional citrate anticoagulation for $\mathrm{LT}$ has been reported by some centres despite most centres not recommending it because of the potential risk of citrate toxicity) ${ }^{6}$ introduces another potential source of variation in outcomes across centres for any multicentre trial. This has implications for trial planning and ensuring adequate sample size to detect a difference in outcomes related to the intervention. Beyond achieving agreement about inclusion and exclusion criteria, any future larger trial will need to achieve "buy in" with respect to the standardized application of evidence-based IoCRRT for those patients randomized to receive it.

Despite these challenges, we agree with the INCEPTION trial's conclusion that a larger trial is feasible despite failing to achieve its intended enrollment target. As the authors suggest, a large, pre-existing network of LT centres, such as United Organ Sharing Network might provide a platform for a multicentre trial. ${ }^{14}$ The need for a large multicentre trial to assess IoCRRT for LT is clear and INCEPTION has taken us one step closer to it. Achieving better evidence regarding the use of IoCRRT for $\mathrm{LT}$ is important as the intervention has clear mechanisms 


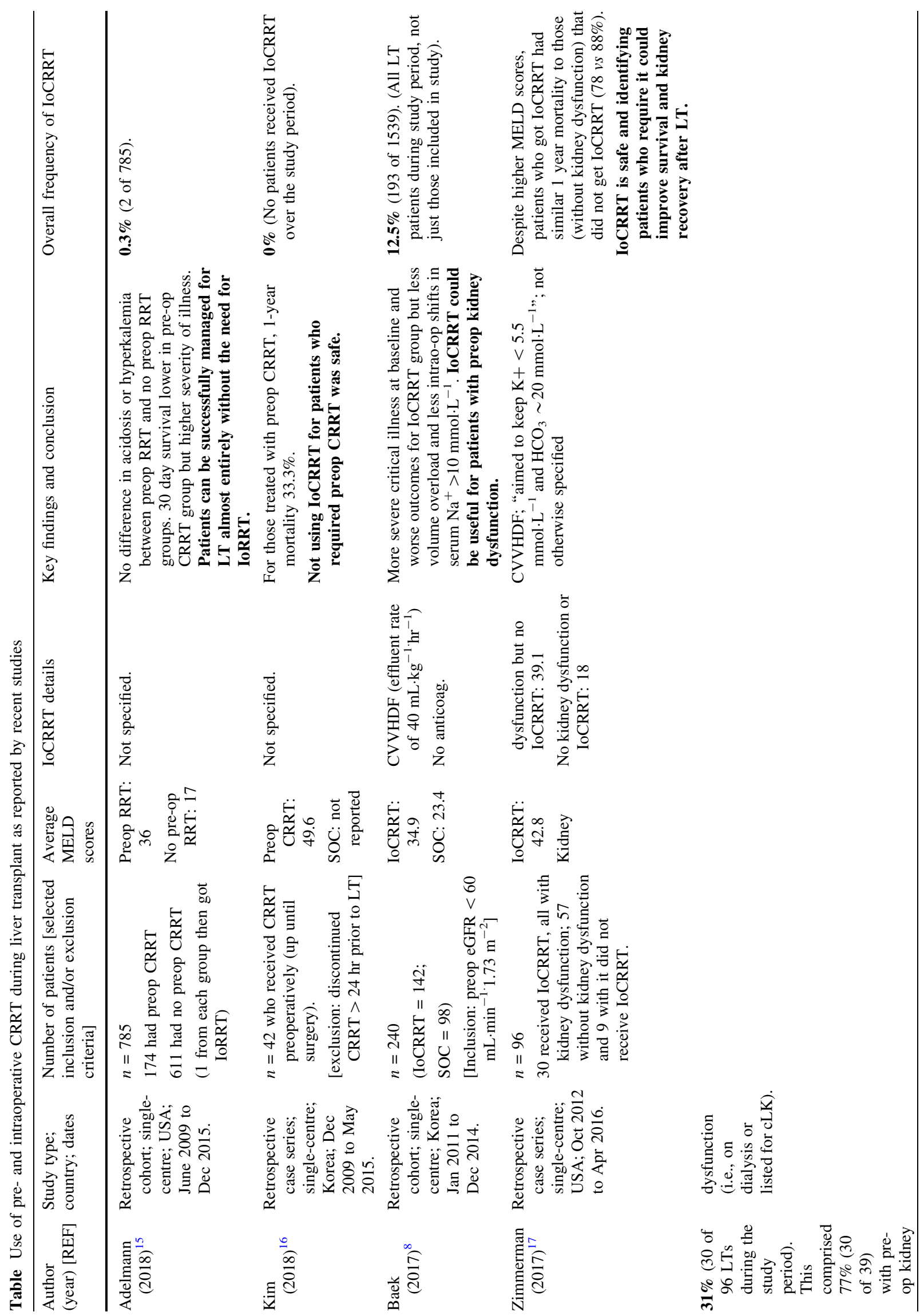




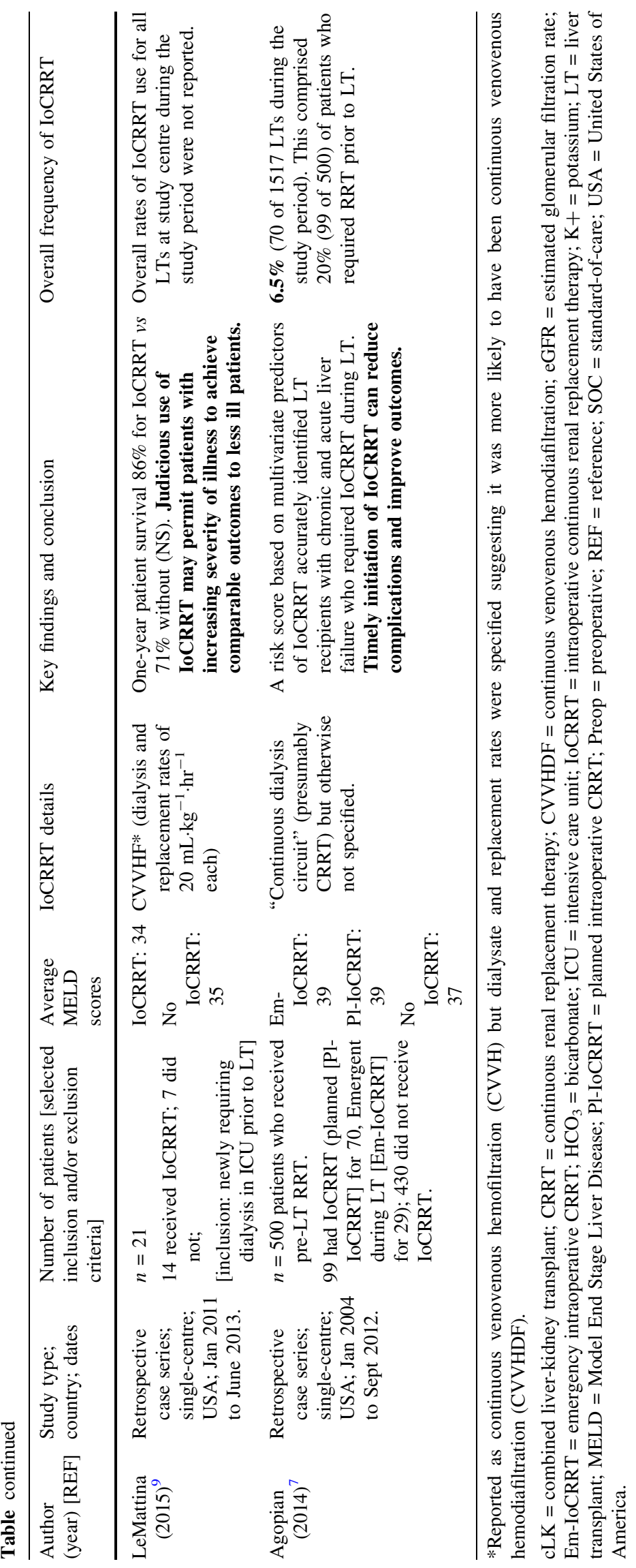


by which it could benefit or harm LT patients. IoCRRT is also expensive and its application appears to vary widely across centres (Table). Addressing the opportunities and challenges presented by the wide variation in current practice will be important to the success of a future, large multicentre trial. We commend the authors of INCEPTION for pushing us all closer to being able to awaken to better evidence for the application of IoCRRT in LT.

\section{INCEPTION: est-ce qu'une étude de plus grande envergure pour évaluer le traitement substitutif peropératoire de l'insuffisance rénale chez des patients subissant une greffe hépatique pourrait devenir réalité?}

La prise en charge périopératoire des patients subissant une greffe hépatique est complexifiée par de nombreux facteurs. Les patients subissant une greffe hépatique sont souvent hémodynamiquement instables et souffrent de troubles métaboliques, de coagulopathies et de défaillance multiviscérale, notamment d'insuffisance rénale aiguë (IRA). ${ }^{1-3}$ La chirurgie en soi peut exacerber ces complications à cause d'importants saignements, de la reperfusion du greffon, et de la perfusion d'importante quantité de liquides intraveineux entraînant des changements rapides de l'équilibre acido-basique (par ex. une acidose), des concentrations d'électrolytes sériques (par ex. une hyperkaliémie ou hyponatrémie), et une hypotonie. L'instabilité hémodynamique et l'œdème cérébral (dû à une pression intracrânienne accrue) associés peuvent également provoquer des complications telles que de l'arythmie, une myélinolyse centropontine, un engagement du tronc cérébral et un échec de la greffe - des résultats finaux bien éloignés de l'objectif premier d'une chirurgie réalisée avant tout afin de restaurer le bon fonctionnement d'un organe. ${ }^{4,5}$

De nombreux centres ont rapporté qu'en administrant un traitement substitutif peropératoire de l'insuffisance rénale pendant une greffe hépatique, l'incidence de ces complications potentielles pouvait être réduite. ${ }^{6-10}$ Diverses modalités de traitement substitutif de l'insuffisance rénale ont été utilisées dans un tel contexte. ${ }^{11}$ Le traitement substitutif peropératoire de l'insuffisance rénale demeure toutefois onéreux, ${ }^{7,12}$ en plus de nécessiter une expertise locale, et pourrait lui aussi être associé à certaines complications, comme celles liées à l'insertion d'un cathéter pour l'hémodialyse temporaire (par ex. infection, pneumothorax), aux troubles métaboliques liés au traitement substitutif de l'insuffisance rénale en continu (par ex. une hypo- ou hypercalcémie, une hypophosphatémie), et aux arythmies qui en découlent. ${ }^{13}$ Ainsi, nous ne pouvons déterminer si le traitement substitutif peropératoire de l'insuffisance rénale devait être utilisé comme traitement de routine pendant une greffe hépatique et, si tel est le cas, pour quels patients. Instinctivement, les patients les plus gravement malades atteints d'une IRA préexistante devraient être ceux bénéficiant le plus probablement de ce type de traitement. En particulier, les patients nécessitant déjà un traitement substitutif de l'insuffisance rénale avant une greffe hépatique pourraient en profiter, car cela leur permettrait d'éviter un phénomène de 'rebond post-dialyse' (soit une exacerbation de l'acidose et une hyperkaliémie) provoqué par l'interruption du traitement substitutif de l'insuffisance rénale avant la greffe hépatique. ${ }^{6}$

Dans ce numéro du Journal, Karvellas et coll., de l'Université de l'Alberta, rapportent les résultats d'une étude pilote monocentrique randomisée contrôlée portant sur un traitement substitutif peropératoire continu de l'insuffisance rénale chez les patients subissant une greffe hépatique, l'étude INCEPTION. ${ }^{14}$ Les patients étaient inclus s'ils souffraient au moins d'une insuffisance rénale légère de base due soit à une IRA (catégorie $\mathrm{R}$ ou pire selon la classification RIFLE) ou d'une insuffisance rénale chronique (taux de filtration glomérulaire estimé $<60$ $\mathrm{mL} \cdot \min ^{1} \cdot 1,73 \mathrm{~m}^{-2}$ ). Après le recrutement de 60 patients, dont $32(53 \%)$ ont été randomisés à recevoir un traitement substitutif peropératoire continu de l'insuffisance rénale ou les soins habituels, l'étude a été interrompue en raison du recrutement trop lent et du manque de fonds. Parmi les 17 patients randomisés à recevoir les soins habituels, sept (41\%) ont changé de groupe et ont reçu un traitement substitutif. Globalement, aucun événement indésirable n'a été rapporté suite au traitement substitutif en soi, et aucune différence significative n'a été observée entre les groupes en matière de résultats cliniques (ce qui n'était toutefois pas le but premier de cette étude, étant donné son manque de puissance pour le faire). La survie globale à un an était excellente $(92 \%$ après l'exclusion des patients n'ayant pas subi de greffe hépatique après avoir été randomisés). Les auteurs concluent qu'une étude multicentrique d'envergure sur le traitement substitutif peropératoire continu de l'insuffisance rénale serait faisable et sécuritaire. ${ }^{14}$

La démarche d'obtenir de meilleures données probantes concernant l'application de ce type de traitement pour la greffe hépatique en réalisant une étude randomisée 


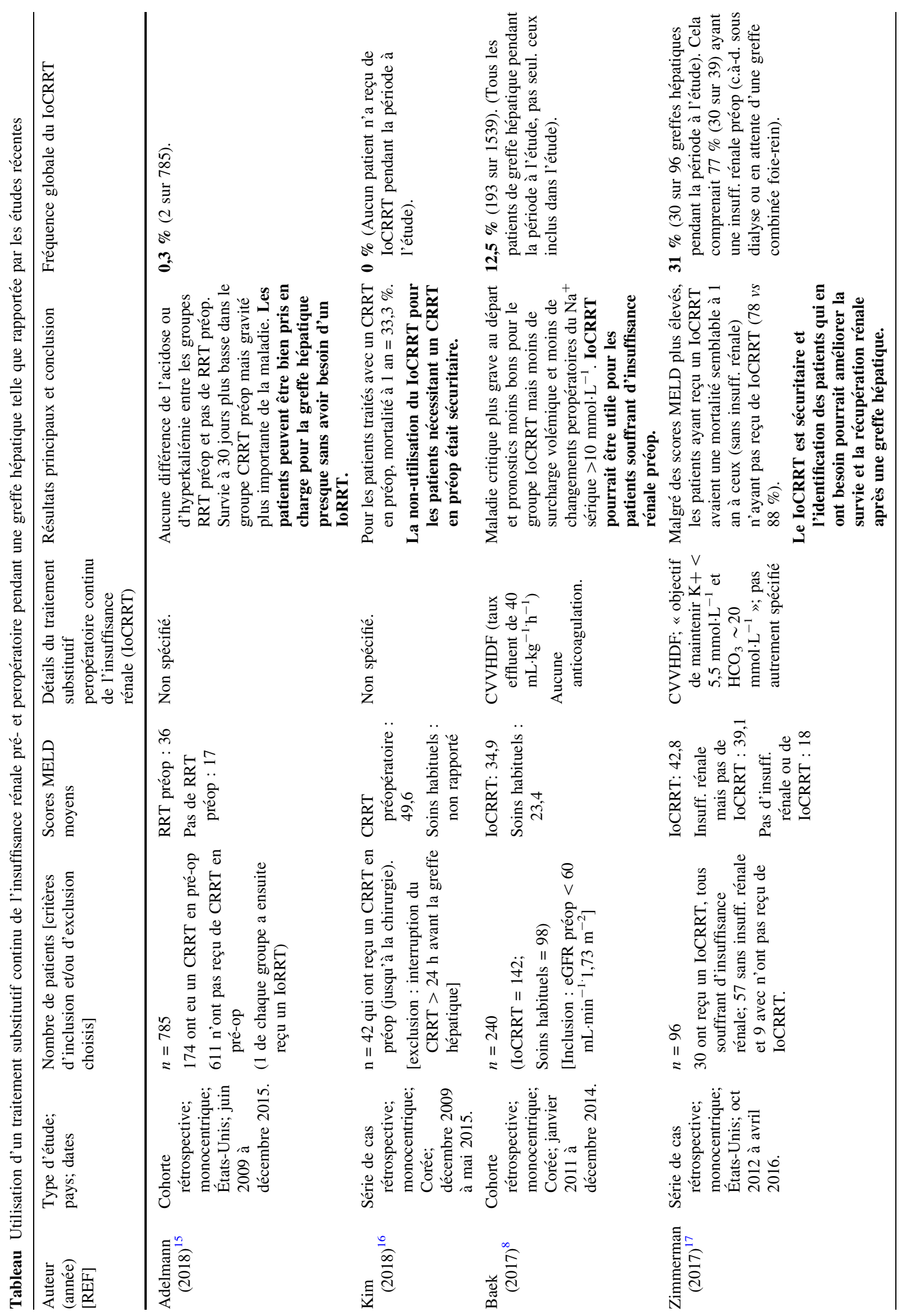




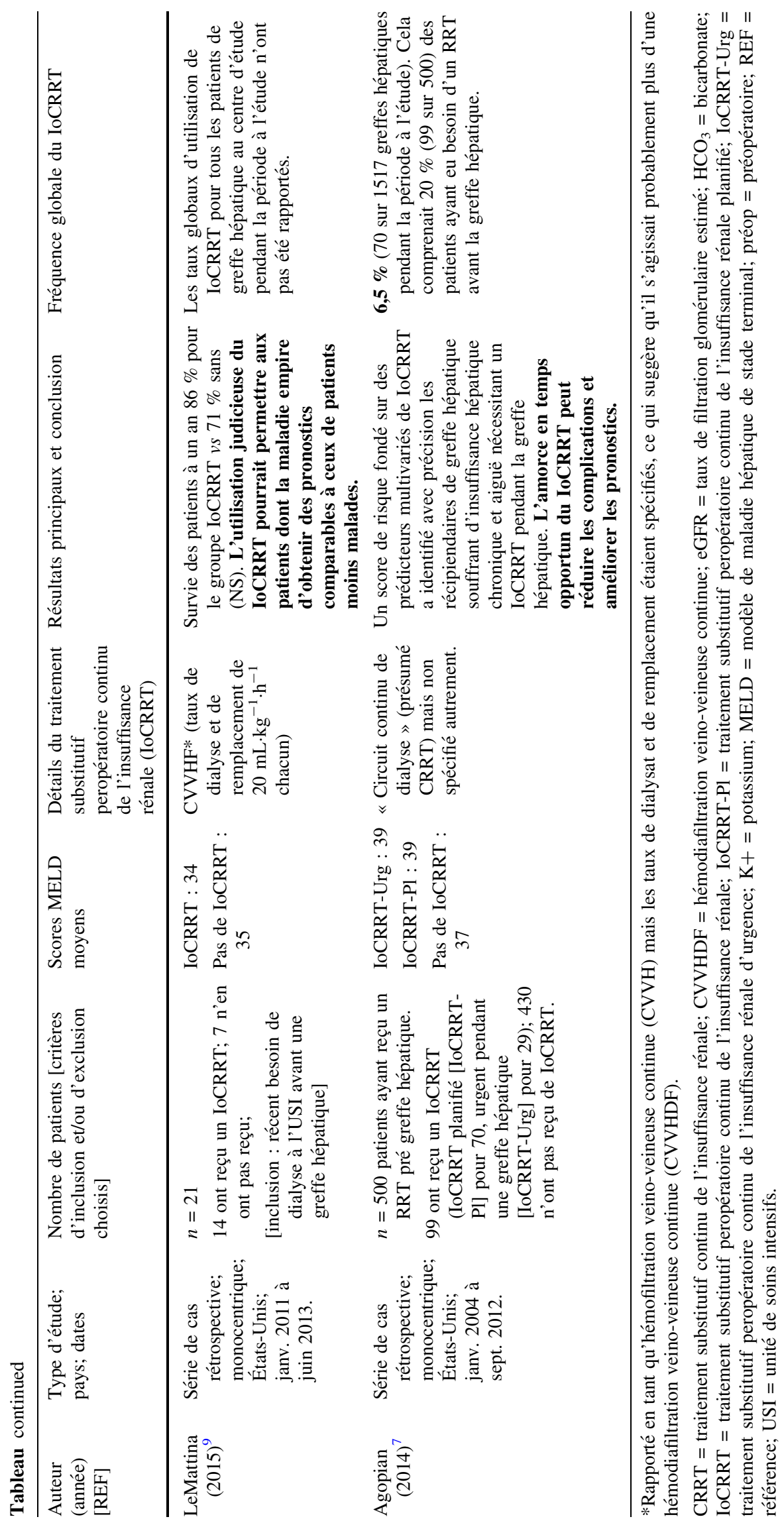


contrôlée d'envergure est louable - si le traitement s'avère efficace, son utilisation plus répandue pourrait améliorer les pronostics des patients. S'il n'est pas efficace, son utilisation réduite pourrait, au minimum, entraîner une diminution importante des coûts de santé. ${ }^{7}$ Un bref compte rendu des études récentes évaluant l'utilisation d'un traitement substitutif de l'insuffisance rénale pré-greffe hépatique et/ou d'un traitement substitutif peropératoire

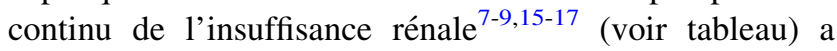
révélé une grande variation en ce qui a trait à la fréquence à laquelle le traitement substitutif peropératoire continu de l'insuffisance rénale est utilisé chez les patients recevant une greffe hépatique. Fait important, il suggère que l'utilisation (ou la non-utilisation) de ce traitement chez les patients atteints ou non d'insuffisance rénale importante en période préopératoire s'inscrit dans les normes de soins actuelles. Les différences observées dans les taux d'utilisation pourraient, dans une certaine mesure, refléter le type de cas traités dans chaque institution, mais sont également probablement liées aux différences locales en matière de ressources et d'expérience avec le traitement substitutif peropératoire continu de l'insuffisance rénale. En effet, l'application de ce traitement complexe dans la pratique varie grandement d'une institution à l'autre; on peut donc présumer que, en l'absence de données probantes de meilleure qualité que celles déjà existantes, la randomisation de patients devant subir une greffe hépatique et souffrant d'une insuffisance rénale même légère à recevoir ou non ce type de traitement devrait être acceptable pour la plupart des cliniciens et ce, peu importe leurs convictions personnelles quant à son utilité. Cela souligne aussi ce qui pourrait constituer un défi majeur à la réalisation d'une vaste étude multicentrique. Malgré des conclusions contradictoires quant à l'efficacité du traitement substitutif peropératoire continu de l'insuffisance rénale pour les patients de greffe hépatique, les études monocentriques récentes évaluant l'utilisation d'un traitement substitutif préopératoire de l'insuffisance rénale et/ou un traitement substitutif peropératoire continu de l'insuffisance rénale (voir tableau) concluent généralement que la pratique dans les centres d'étude constituent la prise en charge la plus adéquate. Bien que ce phénomène n'est probablement pas unique à ce champ d'étude en particulier, il suggère toutefois que les cliniciens ont vraisemblablement déjà formé leur opinion quant aux effets du traitement substitutif peropératoire continu de l'insuffisance rénale pour les patients de greffe hépatique, ainsi que quant aux groupes de patients qui n'en bénéficieront clairement pas ou qui pourraient en souffrir. Par conséquent, nous assistons à une situation dans laquelle la totalité des données probantes suggère qu'il existe un équilibre clinique (ou équipoise), mais dans laquelle les centres pris individuellement pourraient ne pas s'accorder sur le fait qu'il existe un équilibre (c'est-à-dire, certains centres n'accepteraient pas de randomiser leurs patients à recevoir ou non un traitement substitutif peropératoire de l'insuffisance rénale).

Plusieurs éléments des résultats de l'étude INCEPTION sont utiles pour planifier une étude plus importante. Il faut noter que, dans l'étude INCEPTION, il y a eu d'importants transferts du groupe de soins habituels au groupe recevant le traitement étudié $(41 \%) .{ }^{14} \mathrm{Il}$ faudrait tenir compte de la possibilité qu'un nombre élevé de patients puisse passer du groupe recevant les soins habituels au groupe recevant le traitement substitutif peropératoire de l'insuffisance rénale lors du calcul des tailles d'échantillon pour une étude de plus grande envergure. En effet, ce pourrait être un obstacle au recrutement d'un nombre suffisamment important de patients pour pouvoir réaliser une analyse du projet thérapeutique ayant une puissance assez significative. Cela pourrait également refléter l'absence d'équilibre lors de la randomisation de patients pour lesquels la plupart des médecins seraient d'avis qu'un traitement substitutif peropératoire continu de l'insuffisance rénale serait requis. Ainsi, non seulement pourrait-il être ardu d'enrôler de tels patients, mais le risque de contamination pourrait en plus rendre l'effort de recruter de tels patients peu prudent. Afin de compenser, on pourrait envisager d'inclure des patients avec un score MELD plus bas (Model for End-Stage Liver Disease - le score MELD moyen dans l'étude INCEPTION était de 36). Cependant, la probabilité que ces patients aient besoin (et donc bénéficieraient) d'un traitement substitutif peropératoire continu de l'insuffisance rénale en premier lieu est moins élevée, et ils pourraient, en fait, pâtir de recevoir un traitement substitutif de l'insuffisance rénale non nécessaire. ${ }^{18}$ Il faut garder à l'esprit que les complications liées au cathéter de dialyse ainsi que les autres complications d'un traitement substitutif de l'insuffisance rénale en continu (par ex., une instabilité hémodynamique pendant un traitement substitutif de l'insuffisance rénale) peuvent potentiellement avoir un impact négatif sur le pronostic. ${ }^{19}$ Le nombre plus restreint que prévu de patients recrutés dans cette étude pilote n'est pas si surprenant et nous offre une estimation plus réaliste du nombre de centres et du temps nécessaires à réaliser une étude concluante. Globalement, il est encourageant que les résultats de l'étude pilote soient très rassurants en matière de résultats d'innocuité : aucun événement indésirable secondaire au traitement substitutif peropératoire continu de l'insuffisance rénale n'a été rapporté. Il convient de mentionner que le groupe de l'Université de l'Alberta à l'origine de cette étude possède une vaste expertise dans l'utilisation du traitement substitutif peropératoire continu de l'insuffisance rénale; ceci explique peut-être le nombre relativement restreint d'événements indésirables, nombre 
plus bas que ce qui pourrait être anticipé dans une vaste étude multicentrique - ce résultat d'innocuité pourrait par conséquent ne pas être généralisable à d'autres centres. Par exemple, les membres de ce groupe avaient précédemment rapporté une étude d'assurance de la qualité qui était parvenue à minimiser les augmentations périopératoires trop rapides de sodium chez les patients subissant une greffe hépatique. ${ }^{20}$ Plus spécifiquement, ils ont mis au point et introduit des ordonnances standardisées pour amorcer et ajuster le traitement substitutif peropératoire continu de l'insuffisance rénale pendant une greffe hépatique. Ces ordonnances standardisées ont été utilisées pour gérer de façon spécifique une modification adaptée de quantités de liquides utilisées pour le traitement substitutif de l'insuffisance rénale afin de prévenir une correction trop rapide de l'hyponatrémie chez des patients de greffe hépatique, ce qui pourrait avoir prévenu les événements indésirables liés à une correction trop rapide de l'hyponatrémie en cas de traitement substitutif peropératoire continu de l'insuffisance rénale.

Un défi plus général lié à toute étude évaluant l'application d'un traitement substitutif de l'insuffisance rénale en continu chez des patients en état critique réside dans l'hétérogénéité de la pratique de ce type de traitement et l'absence d'étalons validés en ce qui touche à la qualité $\mathrm{du}$ traitement substitutif de l'insuffisance rénale en continu. $^{22,23}$ En raison des variations dans l'application $\mathrm{du}$ traitement substitutif peropératoire continu de l'insuffisance rénale entre les différents centres (par ex., l'utilisation d'une anticoagulation au citrate pour la greffe hépatique a été rapportée par certains centres, malgré le fait que la plupart des centres ne recommandent pas cette pratique en raison du risque potentiel de toxicité au citrate) ${ }^{6}$ toute étude multicentrique comporte donc d'autres sources potentielles de variation dans les résultats obtenus d'un centre à l'autre. Cela a un impact sur la planification de l'étude et la garantie d'une taille d'échantillon adéquate afin de déceler une différence de résultat liée à l'intervention. Outre le fait de se mettre d'accord sur les critères d'inclusion et d'exclusion, toute étude future de plus grande envergure devra réussir à convaincre en ce qui touche à l'application standardisée d'un traitement substitutif peropératoire continu de l'insuffisance rénale fondé sur des données probantes pour les patients randomisés à recevoir ce traitement.

Malgré tous ces défis, nous sommes d'accord avec la conclusion de l'étude INCEPTION: une étude plus importante est faisable, malgré le fait qu'INCEPTION n'ait pas réussi à atteindre sa cible de recrutement. Comme les auteurs le suggèrent, un important réseau préexistant de centres pratiquant la greffe hépatique, tel que le réseau américain UNOS (United Network for Organ Sharing), pourrait constituer une plateforme utile pour réaliser une étude multicentrique. ${ }^{14}$ Le besoin d'une étude multicentrique d'envergure pour évaluer le traitement substitutif peropératoire continu de l'insuffisance rénale pour la greffe hépatique est clair, et l'étude INCEPTION nous mène un peu plus près de cet objectif. Il est important d'obtenir de meilleures données probantes quant à l'utilisation de ce traitement dans cette application : en effet, cette intervention dispose de mécanismes clairs par lesquels elle pourrait être soit bénéfique, soit nocive pour les patients de greffe hépatique. Le traitement substitutif peropératoire continu de l'insuffisance rénale est également onéreux et son application semble varier considérablement d'un centre à un autre (voir tableau). Il sera important d'aborder les possibilités et les défis que présente cette variation significative dans la pratique actuelle afin de garantir la réussite d'une étude multicentrique d'envergure à l'avenir. Nous félicitons les auteurs de l'étude INCEPTION et les remercions de nous avoir rapprochés de données probantes de meilleure qualité pour l'application du traitement substitutif peropératoire continu de l'insuffisance rénale pour la greffe hépatique.

Acknowledgements Edward G. Clark and Swapnil Hiremath are supported by the Department of Medicine, The Ottawa Hospital and University of Ottawa.

Disclosures Edward G. Clark and Swapnil Hiremath have worked on unrelated projects and publications with the senior author of the INCEPTION trial (SMB) within the past five years.

Conflicts of interest None declared.

Editorial responsibility This submission was handled by Dr. Philip M. Jones, Associate Editor, Canadian Journal of Anesthesia.

Remerciements Edward G. Clark et Swapnil Hiremath sont soutenus par le Département de médecine de l'Hôpital d'Ottawa et l'Université d'Ottawa.

Déclarations Edward G. Clark et Swapnil Hiremath ont travaillé sur d'autres projets et publications sans lien avec l'auteur principal de l'étude INCEPTION (SMB) au cours des cinq dernières années.

\section{Conflit d'intérêt Aucun.}

Responsabilité éditoriale Cet article a été traité par Dr Philip M. Jones, rédacteur adjoint, Journal canadien d'anesthésie.

\section{References}

1. Thuluvath PJ, Guidinger MK, Fung JJ, Johnson LB, Rayhill SC, Pelletier SJ. Liver transplantation in the United States, 19992008. Am J Transplant 2010; 10: 1003-19.

2. Matuszkiewicz-Rowinska J, Wieliczko M, Malyszko J. Renal replacement therapy before, during, and after orthotopic liver transplantation. Ann Transplant 2013; 18: 248-55. 
3. Lau C, Martin P, Bunnapradist S. Management of renal dysfunction in patients receiving a liver transplant. Clin Liver Dis 2011; 15: 807-20.

4. Crivellin C, Cagnin A, Manara R, et al. Risk factors for central pontine and extrapontine myelinolysis after liver transplantation: a single-center study. Transplantation 2015; 99: 1257-64.

5. Hilmi I, Horton CN, Planinsic RM, et al. The impact of postreperfusion syndrome on short-term patient and liver allograft outcome in patients undergoing orthotopic liver transplantation. Liver Transpl 2008; 14: 504-8.

6. Matuszkiewicz-Rowinska J, Malyszko J, Wieliczko M. Renal support during liver transplantation: when to consider it? Transplant Proc 2013; 45: 3157-62.

7. Agopian VG, Dhillon A, Baber J, et al. Liver transplantation in recipients receiving renal replacement therapy: outcomes analysis and the role of intraoperative hemodialysis. Am J Transplant 2014; 14: 1638-47.

8. Baek SD, Jang M, Kim W, et al. Benefits of intraoperative continuous renal replacement therapy during liver transplantation in patients with renal dysfunction. Transplant Proc 2017; 49: 1344-50.

9. LaMattina JC, Kelly PJ, Hanish SI, et al. Intraoperative continuous veno-venous hemofiltration facilitates surgery in liver transplant patients with acute renal failure. Transplant Proc 2015; 47: 1901-4.

10. Douthitt L, Bezinover D, Uemura T, et al. Perioperative use of continuous renal replacement therapy for orthotopic liver transplantation. Transplant Proc 2012; 44: 1314-7.

11. Alvarez G, Chrusch C, Hulme T, Posadas-Calleja JG. Renal replacement therapy: a practical update. Can J Anesth 2019; 66: 593-604.

12. Manns B, Doig CJ, Lee $H$, et al. Cost of acute renal failure requiring dialysis in the intensive care unit: clinical and resource implications of renal recovery. Crit Care Med 2003; 31: 449-55.

13. Akhoundi A, Singh B, Vela M, et al. Incidence of adverse events during continuous renal replacement therapy. Blood Purif 2015; 39: 333-9.

14. Karvellas CJ, Taylor S, Bigam D, et al. Intraoperative continuous renal replacement therapy during liver transplantation: a pilot randomized controlled trial (INCEPTION). Can J Anesth 2019; DOI: https://doi.org/10.1007/s12630-019-01454-0.

15. Adelmann D, Olmos A, Liu LL, et al. Intraoperative management of liver transplant patients without the routine use of renal replacement therapy. Transplantation 2018; 102: e229-35.

16. Kim HY, Lee JE, Ko JS, Gwak MS, Lee SK, Kim GS. Intraoperative management of liver transplant recipients having severe renal dysfunction: results of 42 cases. Ann Surg Treat Res 2018; 95: 45-53.

17. Zimmerman MA, Selim M, Kim J, et al. Outcome analysis of continuous intraoperative renal replacement therapy in the highest acuity liver transplant recipients: a single-center experience. Surgery 2017; 161: 1279-86.

18. Clark EG, Bagshaw SM. Unnecessary renal replacement therapy for acute kidney injury is harmful for renal recovery. Semin Dial 2015; 28: 6-11.

19. Silversides JA, Pinto $R$, Kuint $R$, et al. Fluid balance, intradialytic hypotension, and outcomes in critically ill patients undergoing renal replacement therapy: a cohort study. Crit Care 2014; DOI: https://doi.org/10.1186/s13054-014-0624-8.

20. Romanovsky A, Azevedo LC, Meeberg G, Zibdawi R, Bigam D, Bagshaw SM. Serum sodium shift in hyponatremic patients undergoing liver transplantation: a retrospective cohort study. Ren Fail 2015; 37: 37-44.

21. Shen $B, X u J$, Wang $Y$, Jiang $W$, Teng $J$, Ding $X$. Continuous renal replacement therapy quality control and performance measures. Contrib Nephrol 2018; 194: 134-45.

22. Kashani K, Rosner $M H$, Haase $M$, et al. Quality improvement goals for acute kidney injury. Clin J Am Soc Nephrol 2019; 14: 941-53.

23. Rewa OG, Eurich DT, Noel Gibney RT, Bagshaw SM. A modified Delphi process to identify, rank and prioritize quality indicators for continuous renal replacement therapy (CRRT) care in critically ill patients. J Crit Care 2018; 47: 145-52.

Publisher's Note Springer Nature remains neutral with regard to jurisdictional claims in published maps and institutional affiliations. 